\title{
Brush swab as a noninvasive surrogate for tissue biopsies in oral cancer patients to develop clinically translatable epigenetic biomarkers
}

\section{Chi Tonglien Viet ( $\nabla$ cviet@llu.edu )}

Loma Linda University https://orcid.org/0000-0001-5078-7807

Xinyu Zhang

Yale University

$\mathrm{KeXu}$

Yale University

Gary Yu

New York University

Kesava Asam

New York University

Carissa M. Thomas

University of Alabama at Birmingham

Nicholas F. Callahan

University of Illinois at Chicago

Coleen Doan

Loma Linda University

Paul C. Walker

Loma Linda University

Khanh Nguyen

Loma Linda University

Stephanie C. Kidd

Loma Linda University

Steve C. Lee

Loma Linda University

Anupama Grandhi

Loma Linda University

Clint T. Allen

NIDCD: National Institute on Deafness and Other Communication Disorders

Simon Young

University of Texas Health Science Center at Houston School of Dentistry: The University of Texas Health Science Center at Houston School of Dentistry

James C. Melville

University of Texas Health Science Center at Houston School of Dentistry: The University of Texas Health Science Center at Houston School of Dentistry

Jonathan W. Shum

University of Texas Health Science Center at Houston School of Dentistry: The University of Texas Health Science Center at Houston School of Dentistry

Dan T. Viet

Rocky Vista University

Alan S. Herford

Loma Linda University

Dylan F. Roden

Rutgers New Jersey Medical School

Manuel Lora Gonzalez

University of Alabama at Birmingham

Bradley E. Aouizerat

New York University

\section{Research Article}

Keywords: methylation biomarker, oral cancer, head and neck cancer, epigenetic biomarker, brush swab, brush biopsy, biomarker, MethylCap-Seq, methylation array

Posted Date: July 27th, 2021 
DOI: https://doi.org/10.21203/rs.3.rs-653431/v2

License: (c) (i) This work is licensed under a Creative Commons Attribution 4.0 International License. Read Full License 


\section{Abstract \\ Background}

Oral squamous cell carcinoma (OSCC) has poor survival rates. There is a pressing need to develop more precise risk assessment methods to tailor clinical treatment. Epigenome-wide association studies in OSCC have not produced a viable biomarker. These studies have relied on methylation array platforms, which are limited in their ability to profile the methylome. In this study, we use MethylCap-Seq (MC-Seq), a comprehensive methylation quantification technique, and brush swab samples, to develop a noninvasive, readily translatable approach to profile the methylome in OSCC patients.

\section{Methods}

Three OSCC patients underwent collection of cancer and contralateral normal tissue and brush swab biopsies, totaling 4 samples for each patient. Epigenome-wide DNA methylation quantification was performed using the SureSelectXT Methyl-Seq platform. DNA quality and methylation site resolution were compared between brush swab and tissue samples. Correlation and methylation value difference were determined for brush swabs vs. tissues for each respective patient and site (i.e., cancer or normal). Correlations were calculated between cancer and normal tissues and brush swab samples for each patient to determine the robustness of DNA methylation marks using brush swabs in clinical biomarker studies.

\section{Results}

There were no significant differences in DNA yield between tissue and brush swab samples. Mapping efficiency exceeded $90 \%$ across all samples, with no differences between tissue and brush swabs. The average number of CpG sites with at least 10x depth of coverage was 2,716,674 for brush swabs and 2,903,261 for tissues. Matched tissue and brush swabs had excellent correlation ( $r=0.913$ for cancer samples and $r=0.951$ for normal samples). The methylation profile of the top 1,000 CpGs was significantly different between cancer and normal samples (mean p-value $=0.00021)$ but not different between tissues and brush swabs (mean p-value $=0.11$ ).

\section{Conclusions}

Our results demonstrate that MC-Seq is an efficient platform for epigenome profiling in cancer biomarker studies, with broader methylome coverage than array-based platforms. Brush swab biopsy provides adequate DNA yield for MC-Seq, and taken together, our findings set the stage for development of a noninvasive methylome quantification technique for oral cancer with high translational potential.

\section{Introduction}

Each year 30,000 patients are diagnosed with oral cavity squamous cell carcinoma (OSCC), and unfortunately the incidence is on the rise. [1-3]. Even for these early stage patients, the five-year survival rate is $60 \%$ [4]. Poor survival rates are in part due to inaccurate risk prediction. Early stage $0 S C C$ is primarily treated with surgical resection of the cancer, with or without adjuvant treatments such as an elective lymphadenectomy, radiation, or chemoradiation, for patients with high risk features. Currently, risk prediction to assign adjuvant treatment is entirely based on clinicopathologic information. Multiple retrospective and prospective studies have shown that these standard clinicopathologic factors have moderate accuracy with a concordance statistic (c-statistic) of 0.7 [4, 5]. The key to improving survival in OSCC lies in developing more accurate risk prediction methods, particularly in early stage patients. Although OSCC is a heavily epigenetically-regulated cancer [6], optimizing risk prediction using methylation features remains in its infancy. Methylation is one of the most frequent epigenetic changes in early oral carcinogenesis that is linked to cancer progression [6]. While several methylation studies in 0SCC patients [6-16], including our own studies [7, 8], have highlighted differential methylation features between low and high risk patients, none of these studies have resulted in a clinically meaningful biomarker. Two main shortcomings of these previous studies are: 1) failure to use a clinically translatable array platform, and 2) failure to quantify methylation in real time, as cancer treatment is occurring.

With respect to the first challenge, the vast majority of methylation array studies in OSCC have used array-based platforms. While the Illumina Methylation $450 \mathrm{~K}$ or EPIC array are the most commonly used platforms for epigenome-wide association studies (EWAS), CpG site quantification is restricted at an upper limit of 870,000 sites, and results from these platforms have not been converted into a clinically-accessible risk prediction tool. Furthermore, the EPIC array content is frequently updated to enrich for cancer-associated genes, making comparison across cohorts challenging. Methylation capture sequencing (MCseq) has a scalable workflow that can quantify methylation in a small subset of genes or the entire genome using next generation sequencing (NGS), with a higher likelihood of clinical translation due to broader CpG coverage in a more agnostic manner while maintaining its resolution in samples with modest DNA quantities [17].

With respect to the second challenge, clinical translation of a biomarker requires measurement at the onset of treatment in order to determine risk and the need for treatment escalation. Waiting until after cancer removal for the formalin-fixed, paraffin-embedded (FFPE) tissues would limit clinical translatability. The oral cavity has the advantage of being readily accessible for sampling, not only with tissue biopsies, but also with noninvasive techniques. Herein, we determine methylation features using noninvasive brush swabs.

In this study, we hypothesize that brush swab biopsies serve as a robust noninvasive method to quantify cancer-specific methylation features. Using tissue and brush swab biopsies collected from OSCC patients at the time of surgery: 1) we determine the concordance between the methylation signature of cancer 
tissues and swabs vs. matched normal tissues and swabs using MC-Seq, and 2) we establish a workflow in which brush swabs and MC-seq are used at the time of diagnosis to establish a methylation signature that can be used to determine risk of mortality.

\section{Methods}

\section{Patient selection and data collection}

The patients were enrolled in a multi-institutional prospective clinical study in which biological samples and clinicopathologic information were collected. Collection of clinical data and samples was approved by the Institutional Review Board at each institution, which included Loma Linda University (LLU), University of Illinois Chicago (UIC), and University of Alabama at Birmingham (UAB). Patients were eligible if they were $\geq 18$ years of age, had biopsy-proven squamous cell carcinoma of oral cavity sub-sites, including oral tongue, maxillary and mandibular gingiva, hard palate, floor of mouth, buccal mucosa, and lip mucosa, and no previous treatment of OSCC. Clinical and pathologic stages were recorded based on the American Joint Committee on Cancer (AJCC) Eighth Edition Staging Manual [18]. We collected the following information from the chart review: age, sex, race, smoking and alcohol use, staging, tumor location, pathologic characteristics, and treatment modalities received in addition to tumor ablation. Biological samples collected at the time of surgery include flashfrozen cancer and contralateral normal tissue, and brush swab biopsies of the cancer and contralateral normal site. Samples were stored in $-80^{\circ} \mathrm{C}$. A total of 3 patients were randomly chosen from the ongoing prospective clinical study for the current study.

\section{Nucleic acid extraction and sample preparation}

DNA was extracted from the fresh-frozen tissue and brush swabs of the cancer and contralateral normal side of 3 patients, totaling 12 samples (4 samples per patient). Genomic DNA quality was determined by spectrophotometry and concentration was determined by fluorometry. DNA integrity and fragment size were determined using a microfluidic chip run on an Agilent Bioanalyzer.

\section{MC-seq target enrichment library prep}

Indexed paired-end whole-genome sequencing libraries were prepared using the SureSelect XT Methyl-Seq kit (Agilent). Genomic DNA was sheared to a fragment length of 150-200 bp using the Covaris E220 system. Fragmented sample size distribution was determined using the Caliper LabChip GX system (PerkinElmer). Fragmented DNA ends were repaired with T4 DNA Polymerase and Polynucleotide Kinase and "A" base was added using Klenow fragment followed by AMPure XP bead-based purification (Beckman Coulter). The methylated adapters were ligated using T4 DNA ligase followed by bead purification with AMPure XP. Quality and quantity of adapter-ligated DNA were assessed with the Caliper LabChip GX system. Samples were enriched for targeted methylation sites by using the custom SureSelect Methyl-Seq Capture Library. Hybridization was performed at $65^{\circ} \mathrm{C}$ for $16 \mathrm{~h}$ using a thermal cycler. Once the enrichment was completed, the samples were mixed with streptavidin-coated beads (Thermo Fisher Scientific) and washed with a series of buffers to remove non-specific DNA fragments. DNA fragments were eluted from beads with $0.1 \mathrm{M} \mathrm{NaOH}$. Unmethylated C residues of enriched DNA underwent bisulfite conversion using the EZ DNA Methylation-Gold Kit (Zymo Research). The SureSelect enriched and bisulfite-converted libraries underwent PCR amplification using custom made primers (IDT). Dual-indexed libraries were quantified by quantitative polymerase chain reaction (qPCR) with the Library Quantification Kit (KAPA Biosystems) and inserts size distribution was assessed using the Caliper LabChip GX system.

\section{Flow cell preparation and sequencing}

Samples were sequenced using 100 bp paired-end sequencing on an Illumina HiSeq NovaSeq according to Illumina protocol. A positive control (prepared bacteriophage Phi X library) was added into every lane at a concentration of $0.3 \%$ to assess sequencing quality in real time.

\section{Preprocessing and quality control}

Signal intensities were converted to individual base calls during each run using the system's Real Time Analysis software. Sample de-multiplexing was performed using Illumina's CASAVA 1.8 .2 software suite. The sample error rate was required to be less than $1 \%$ and the distribution of reads per sample in a lane to be within reasonable tolerance. Sequence data quality were examined using FastQC (ver. 0.11.8). Adapter sequences and fragments with poor quality were removed by Trim_galore (ver. 0.6.3_dev). Bismark pipelines (ver. v0.22.1_dev) were used to align the reads to the bisulfite human genome (hg19) with default parameters.[19] Sample alignment to the human genome was performed using bowtie 2 (ver. 2.3.5.1). Quality-trimmed paired-end reads were converted into a bisulfite forward (C->T conversion) or reverse (G->A conversion) strand read. Duplicated reads were removed from the Bismark mapping output and $\mathrm{CpG}$ extracted. All $\mathrm{CpG}$ sites were grouped by sequencing coverage (i.e., read depth); CpG sites with coverage $\geq 10 \mathrm{x}$ depth were retained for analysis to ensure high MC-Seq data quality. Genes were annotated using Homer annotatePeaks.pl.

\section{Comparison of methylation between tissue and brush swab biopsies}

Pearson correlations were calculated between tissue and brush biopsy samples of matched anatomic sites. Pearson correlation and absolute difference were calculated among common CpG sites between the matched tissue and brush biopsies. Scatterplots were rendered showing the correlation of $\beta$ values from all CpG sites measured by MC-sEq. Separate scatterplots were rendered showing the concordance of these $\mathrm{CpG}$ sites between tissues and brush swabs for the cancer sites and the normal sites. Student t-tests were performed to compare $\beta$ values between cancer and normal groups or tissue and brush swab groups. The most significant 1,000 CpGs features in cancer vs. normal groups were selected. Based on these results the -log10(t-test p-value) was calculated for each of the $1,000 \mathrm{CpG}$ sites to compare the degree of divergence in the significance of the test statistics for these $1,000 \mathrm{CpG}$ between 1) cancer vs. normal and 2) tissue vs. brush swabs.

\section{Statistical analyses}

Statistical analyses were performed in R environment (v. 4.1.0). 


\section{Results}

\section{Patient cohort characteristics and DNA quality}

Clinicopathologic information for the 3 enrolled patients are detailed in Table 1. The 3 patients comprised both early and late stage OSCC (stage I and IV), as well as varying tobacco and alcohol consumption habits. Patients were 49 and 68 years old. Two patients were male and one was female. All patients were white, non-Hispanic. Cancer and contralateral normal tissue and brush swab biopsies collected at the time of surgery underwent DNA extraction, with the yield and quality shown in Table 2. With a total input volume of $30 \mu \mathrm{L}$ for each sample, total input for tissue DNA ranged from $187 \mathrm{ng}$ to $660 \mathrm{ng}$, and an average of $390 \mathrm{ng}$. Total input for swab DNA ranged from 51ng to 1998ng, with an average of 532ng. The input range was consistent with our previous study demonstrating reproducible CpG site quantification using MC-Seq across this range [17].

Table 1

Patient demographic characteristics.

\begin{tabular}{|llllllllll|}
\hline Patient & Age & Sex & Race & Tobacco use, pack years & Alcohol, drinks/wk & Site & TNM & Stage & Grade \\
\hline $\mathbf{1}$ & 68 & F & White & Never & Never & Tongue & T1N0M0 & I & Moderate \\
\hline $\mathbf{2}$ & 68 & M & White & Former, 53 & Former, 24 & Tongue & T4aN0M0 & IV & Moderate \\
\hline $\mathbf{3}$ & 49 & M & White & Current, 72 & Current, 14 & Mandible & T4bN3bM0 & IV & Moderate \\
\hline
\end{tabular}

Table 2

Characteristics of genomic DNA used as input for sequencing of tissue and brush swab biopsies.

\begin{tabular}{|lllllll|}
\hline Sample & DNA concentration $\mathbf{n g} / \boldsymbol{\mu l}$ & $\mathbf{A 2 6 0}$ & $\mathbf{A 2 8 0}$ & $\mathbf{2 6 0 / 2 8 0}$ & $\mathbf{2 6 0 / 2 3 0}$ & gDNA input, ng \\
\hline 1C swab & 3.96 & 0.01 & -0.004 & -2.99 & -0.18 & 118.8 \\
\hline 1C tissue & 22.00 & 4.45 & 2.210 & 2.01 & 2.18 & 660.0 \\
\hline 1N swab & 1.70 & -0.05 & -0.048 & 0.95 & 0.33 & 51.0 \\
\hline 1N tissue & 6.24 & 0.44 & 0.220 & 2.01 & 2.75 & 187.2 \\
\hline 2C swab & 4.24 & 0.01 & -0.027 & -0.27 & -0.09 & 127.2 \\
\hline 2C tissue & 11.40 & 1.00 & 0.463 & 2.16 & 2.85 & 342.0 \\
\hline 2N swab & 6.32 & 0.07 & 0.014 & 5.14 & -1.45 & 189.6 \\
\hline 2N tissue & 8.00 & 0.62 & 0.290 & 2.12 & 3.04 & 240.0 \\
\hline 3C swab & 66.60 & 1.82 & 0.971 & 1.87 & 2.86 & 1998.0 \\
\hline 3C tissue & 21.80 & 2.16 & 1.314 & 1.65 & 0.80 & 654.0 \\
\hline 3N swab & 23.60 & 0.45 & 0.217 & 2.06 & 4.70 & 708.0 \\
\hline 3N tissue & 8.48 & 0.39 & 0.191 & 2.05 & 5.66 & 254.4 \\
\hline
\end{tabular}

\section{MC-Seq mapping efficiency assessment}

Table 3 details the mapping efficiency for each biological sample. Using MC-Seq sequences mapped to the reference genome with an average mapping efficiency of $90 \%$ across all samples. There were no significant differences in mapping efficiency between tissues and brush swab samples (Fig. 1C). The average difference in mapping efficiency between the paired brush swabs and tissues was minimal, at $-0.567 \%$, in favor of tissue samples, with a range of -1.9 to $1.7 \%$. The majority of methylated C's appeared in a CpG context. We graphed the depth of read for each CpG across all queried CpGs and demonstrated an inflection point at $10 x$ coverage (Fig. 1A). This finding was similar to our previous technical validation study, in which the majority of $\mathrm{CpG}$ sites exhibited at least 10x coverage [17]. We therefore applied this cutoff, focusing our analysis on $\mathrm{CpG}$ sites with at least 10x coverage. Average number of CpGs with at least $10 x$ coverage was $2,716,674$ for swab samples and 2,904,261 for tissue samples, with no significant difference between the two sample types, which is in excess of 3-fold greater CpGs interrogated than the most commonly used tool to measure the DNA methylome, the Illumina EPIC array. Figure 1B indicates the number of $\mathrm{CpGs}$ with at least 10x coverage for each of the 12 individual samples. 
Table 3

MC-Seq mapping efficiency among tissue and brush swab samples.

\begin{tabular}{|c|c|c|c|c|c|c|c|c|c|c|c|}
\hline Name & $\begin{array}{l}\text { Mapping } \\
\text { efficiency } \\
\text { (\%) }\end{array}$ & $\begin{array}{l}\text { Difference } \\
\text { between } \\
\text { swab and } \\
\text { tissue (\%) }\end{array}$ & $\begin{array}{l}\text { Sequence } \\
\text { pairs } \\
\text { analyzed } \\
\text { in total }\end{array}$ & $\begin{array}{l}\text { Number of } \\
\text { paired-end } \\
\text { alignments } \\
\text { with a } \\
\text { unique } \\
\text { best hit }\end{array}$ & $\begin{array}{l}\text { Duplicate } \\
\text { (\%) }\end{array}$ & $\begin{array}{l}\text { Sequence } \\
\text { pairs after } \\
\text { removing } \\
\text { duplicate }\end{array}$ & $\begin{array}{l}\text { Total } \\
\text { number of C }\end{array}$ & $\begin{array}{l}\text { Total } \\
\text { methylated } \\
\text { C's in CpG } \\
\text { context }\end{array}$ & $\begin{array}{l}\text { Total } \\
\text { methylated } \\
\text { C's in CHG } \\
\text { context }\end{array}$ & $\begin{array}{l}\text { Total } \\
\text { methylated } \\
\text { C's in CHH } \\
\text { context }\end{array}$ & $\begin{array}{l}\text { Cpl } \\
\text { der } \\
\text { col } \\
>11\end{array}$ \\
\hline $\begin{array}{l}1 C \\
\text { swab }\end{array}$ & 90.7 & 0.1 & 42303352 & 38369629 & 52.22 & 18333433 & 749965581 & 37720278 & 2408697 & 5995678 & $27:$ \\
\hline $\begin{array}{l}1 \mathrm{C} \\
\text { tissue }\end{array}$ & 90.6 & & 34732207 & 31480976 & 28.42 & 22534549 & 927088792 & 44803115 & 2908059 & 7258418 & 285 \\
\hline $\begin{array}{l}1 \mathrm{~N} \\
\text { swab }\end{array}$ & 89.3 & -0.7 & 35997131 & 32160165 & 69.49 & 9811824 & 392619063 & 19593941 & 1231170 & 3078167 & 184 \\
\hline $\begin{array}{l}1 \mathrm{~N} \\
\text { tissue }\end{array}$ & 90.4 & & 38013580 & 34357686 & 50.99 & 16840023 & 701455939 & 36205354 & 2320490 & 5789438 & 26 \\
\hline $\begin{array}{l}2 \mathrm{C} \\
\text { swab }\end{array}$ & 91.0 & 1.7 & 49206194 & 44795293 & 54.25 & 20495859 & 825691357 & 41320643 & 2723999 & 6792423 & $28 \%$ \\
\hline $\begin{array}{l}2 \mathrm{C} \\
\text { tissue }\end{array}$ & 89.3 & & 48990997 & 43728505 & 39.71 & 26364632 & 1095985402 & 53089834 & 3494884 & 8728001 & $30 ؟$ \\
\hline $\begin{array}{l}2 \mathrm{~N} \\
\text { swab }\end{array}$ & 86.7 & -1.9 & 40037338 & 34712643 & 48.22 & 17975067 & 745570397 & 35629449 & 2300915 & 5877073 & $26 t$ \\
\hline $\begin{array}{l}2 \mathrm{~N} \\
\text { tissue }\end{array}$ & 88.6 & & 37524719 & 33261053 & 48.50 & 17128931 & 713630671 & 35589419 & 2277861 & 5673182 & $26 \varepsilon$ \\
\hline $\begin{array}{l}3 \mathrm{C} \\
\text { swab }\end{array}$ & 90.7 & -0.8 & 36703056 & 33280675 & 26.89 & 24331566 & 1000848080 & 50517501 & 3160407 & 7841535 & 301 \\
\hline $\begin{array}{l}3 \mathrm{C} \\
\text { tissue }\end{array}$ & 91.5 & & 39728186 & 36357410 & 28.90 & 25848885 & 1069409107 & 52603435 & 3264928 & 8042519 & $31 c$ \\
\hline $\begin{array}{l}3 \mathrm{~N} \\
\text { swab }\end{array}$ & 88.3 & -1.8 & 52334030 & 46226126 & 34.76 & 30156313 & 1221133676 & 62639138 & 3840377 & 9388785 & $32 \%$ \\
\hline $\begin{array}{l}3 \mathrm{~N} \\
\text { tissue }\end{array}$ & 90.1 & & 41553671 & 37445490 & 39.68 & 22588890 & 950370928 & 50055981 & 3072187 & 7486332 & $29 \varepsilon$ \\
\hline $\begin{array}{l}\text { Average } \\
\text { swab }\end{array}$ & 89.45 & -0.567 & 42763517 & 38257422 & 48.00 & 20184010 & 822638026 & 41236825 & 2610928 & 6495610 & 271 \\
\hline $\begin{array}{l}\text { Average } \\
\text { tissue }\end{array}$ & 90.00 & & 40090560 & 36105187 & 39.00 & 21884318 & 909656807 & 45391190 & 2889735 & 7162982 & $29 c$ \\
\hline
\end{tabular}

\section{Distribution of methylome regions}

We determined the distribution of CpG sites profiled by MC-Seq among the CpG sites successfully measured at 10X depth of read or greater overlapping across all 12 samples $(3,566,843 \mathrm{CpGs})$. Figure 1D demonstrates that $36 \%$ were in introns, $26 \%$ were in promoters, $19 \%$ were in exons, and $19 \%$ were in intergenic regions. Overall, MC-Seq provided more robust coverage of functional gene regions in the methylome than typically provided by the EPIC array, detecting ten-fold more $\mathrm{CpG}$ sites in promoter regions and exons than the EPIC array. We determined that $484,697 \mathrm{CpGs}$ from the EPIC array, the majority of which were also found on the $450 \mathrm{~K}(396,409 \mathrm{CpG})$ were profiled by MC-Seq with at least $10 x$ coverage. While the breakdown of these CpGs was $33 \%$ intron, $33 \%$ promoter, $15 \%$ exon, and $19 \%$ intergenic, the total number of $\mathrm{CpGs}$ in the functional gene regions was proportionally lower owing to the more limited coverage (Fig. 1D).

\section{Correlation between brush swab and tissue biopsies from matched anatomic sites}

Overall, the correlation among $\mathrm{CpG}$ site methylation across all samples was high, all exceeding $90 \%$. The average correlation between tissue and brush swabs $(n=12)$ among all CpG sites shared among the entire sample (cancer + control) ( $s=3,566,843)$ was $93.2 \%(95 \%$ confidence interval: $93.23 \%$, 93.25\%). The average correlation between tissue and brush swabs $(n=6)$ among all CpG sites shared among cancer samples was $91.3 \%$ ( $95 \%$ confidence interval: $91.32 \%$, 91.35\%). The average correlation between tissue and brush swabs $(n=6)$ among all $\mathrm{CpG}$ sites shared among normal samples was $95.1 \%(95 \%$ confidence interval: $95.13 \%, 95.14 \%)$. A scatterplot of the $\mathrm{CpGs}$ with 10x coverage was generated for the cancer samples and the normal samples separately, demonstrating high concordance between tissue and brush swabs (Fig. 2A and 2B).

The top methylation features are differentially methylated between cancer and normal samples, but not between tissues and brush swabs

We focused on the top 1,000 most variable methylation features between cancer and normal samples, which would be expected to differ considerably less between tissue and brush swab sampling methods. The $\mathrm{p}$-values for each test of difference in $\mathrm{CpG}$ methylation by $\mathrm{t}$-test were expressed as -log ${ }_{10}(\mathrm{p}$-value), and averaged 3.67 (i.e., $\mathrm{p}=0.00021$ ) between cancer vs. normal. The same $\mathrm{CpG}$ sites were not differentially methylated, with an average -log ${ }_{10}(\mathrm{p}-\mathrm{value})=0.96$ (i.e., $\mathrm{p}=0.11$ ) between tissue vs. brush swabs (Fig. 2 C). The results suggest that brush swabs are a clinically viable surrogate for tissue biopsies. 


\section{Discussion}

MC-seq is a scalable, CLIA-approvable methylation assay that is currently not widely used in cancer research

EWAS studies in cancer patients have identified interindividual variability in the epigenome, and the recent availability of affordable EWAS technologies have led to a rapid increase in epigenetic biomarker studies aimed at identifying differential methylation features that could be predictive of clinical outcome. The most commonly used platforms are array-based, like the Illumina Human 450K and Infinium MethylationEPIC arrays, which provide limited coverage of CpG sites across the epigenome. Whole genome bisulfite sequencing (WGBS) is the most comprehensive method for epigenome profiling, capturing 28 million CpGs. However, the cost, intensive workflow, and need for high quality and quantity of DNA input significantly limit its clinical translatability, particularly in cancer treatment. MC-Seq has emerged as a promising intermediary between arrays and WGBS, using NGS to capture significantly more CpGs than arraybased platforms, while having the advantage of being more high-throughput and affordable than WGBS. We and others have compared CpG coverage and efficiency of different methylation quantification platforms [17, 20,21]. A recent publication from our group has demonstrated that MC-Seq is a more reliable and efficient platform for epigenome profiling than array-based platforms like the EPIC array. When the EPIC array and MC-Seq were compared in peripheral blood mononuclear cell samples, MC-Seq captured significantly more CpGs in coding regions and CpG islands than the EPIC array. The EPIC array captured 846,464 CpG sites per sample, whereas MC-Seq captured 3,708,550 CpG sites per sample. Of the 472,540 CpG sites captured by both platforms, there was high correlation $(r=0.98-0.99)$ in methylation status [17]. Moreover, while the EPIC array is enriched for genes with known roles in carcinogenesis, MC-Seq quantifies methylation in a more agnostic manner and profiles 3-4 times more CpGs than the EPIC array, allowing for a higher chance of discovering novel epigenetic modifications in cancer. Furthermore, the coverage areas within each gene were more comprehensive than the EPIC array and other commonly used methylation analysis techniques, like PCR or pyrosequencing. Herein, we demonstrated that MC-Seq captured significantly more CpG sites within functional gene regions, owing to the higher overall profiling capability of this technique.

\section{Oral SCC is an epigenetically-regulated cancer with promising methylation biomarker candidates}

Methylation studies on OSCC patients [6-16] including our own studies [7, 8] have demonstrated that methylation is a common event and highlighted specific genes for mechanistic studies. For example, a EWAS using the Illumina Human 450K array on 108 head and neck SCC patients of multiple sub-sites including oral cavity identified hypermethylation and inactivation of key tumor suppressor genes [9]. Clinical translation of these methylation biomarker studies has been limited due to: 1) combining OSCC with other head and neck cancer sub-sites (i.e., oropharynyx, hypopharynx, larynx), which creates a heterogeneous cohort that fails to recognize OSCC as a distinct clinical disease, and 2) relying solely on array-based platforms, which query a limited number of CpGs. As a result, none of these studies have produced a methylation biomarker with high prognostic performance.

In addition to being a distinct clinical subsite from other head and neck sites, the oral cavity is an easily accessible anatomic site for non-invasive biopsy techniques. Clinical translation of a biomarker requires that it can be measured during treatment. Waiting until after tumor removal for the formalin-fixed, paraffin-embedded (FFPE) tissues delays potentially necessary treatment. Researchers have used both saliva and brush swabs to noninvasively sample OSCC cells at the time of diagnosis. In our own studies, we have used saliva to identify methylation biomarkers of OSCC. We demonstrated that a multi-gene panel could be constructed using either a methylation array or Methylight, a polymerase chain reaction (PCR) technique [7, 8]. However, we and others have shown that concordance of methylation between saliva and cancer tissue is highly variable [22, 23].

\section{Brush swabs and MC-Seq represent a noninvasive method to quantify methylation biomarkers}

Our approach of using brush swabs and MC-Seq to determine the methylation signature at the time of diagnosis has a high potential for clinical translatability. We demonstrated in this study that brush swab and tissue biopsies from matched sites had highly correlated methylation signatures. Furthermore, the DNA quality and quantity from brush swab samples were adequate to perform MC-SEq. Mapping efficiency was equivalent between tissues and brush swabs. Given the high correlation between the paired tissues and brush swabs, and the satisfactory DNA yield, brush swabs could serve as a clinically robust surrogate to tissue biopsies. One previous study has assessed the reliability of brush swab DNA for MC-Seq compared to the Human 450K array [20], drawing similar conclusions to our study [17] that MC-Seq offered broader coverage of CpG sites and that sample-based correlation was high ( $r=$ 0.98) between the two platforms. However, they did not compare brush swab to underlying tissue collection. To our knowledge our study represents the first to directly compare the epigenome-wide signature of matched brush swabs and tissues, with the results having important implications in OSCC biomarker research.

\section{Conclusions}

Our study establishes a workflow for a large-scale clinical study using brush swab samples and MC-Seq to noninvasively determine the methylation signature of OSCC patients at the time of diagnosis, which could be used to establish risk stratification schemes.

\section{Abbreviations}

EWAS: epigenome-wide association study; MC-Seq: MethylCap-Seq; OSCC: oral squamous cell carcinoma

\section{Declarations}


Ethics approval and consent to participate: Institutional Review Board approval was obtained to collect biological samples and create the de-identified patient databases at each respective institution.

Consent for publication: Not applicable.

Availability of data and materials: All data generated for this study are included in this article.

Competing interests: Not applicable.

Funding: The study is supported by National Institute on Drug Abuse (R01DA047063 and R01DA047820), National Institute of Dental and Craniofacial Research (K23DE030250) and the Oral Maxillofacial Surgery Foundation.

Authors' contributions: CTV and BEA designed the study, wrote the manuscript, and coordinated enrollment of the multiple institutions into the studies. $\mathrm{XZ}$, KX, GY and KA performed analysis of the data. CMT, NFC, PCW, KN, SCK, SCL, CTA, SY, ASH, DFR coordinated Institutional Review Board approval, patient enrollment and database creation at their respective sites. CD, JCM, JWS, AG completed the patient databases and performed retrospective chart reviews at their respective institutions. DTV managed the de-identified database. All authors edited the manuscript.

Acknowledgements: The authors appreciate the support of the Yale Center of Genomic Analysis. We would also like to thank the Women of Head and Neck Reconstructive Surgery (HERS) Consortium.

\section{References}

1. Gulland A. Oral cancer rates rise by two thirds. BMJ. 2016;355:i6369.

2. Tota JE, Anderson WF, Coffey C, Califano J, Cozen W, Ferris RL, St John M, Cohen EE, Chaturvedi AK, Rising incidence of oral tongue cancer among white men and women in the United States, 1973-2012. Oral Oncol, 2017. 67: p. 146-152.

3. Kim YJ, Kim JH. Increasing incidence and improving survival of oral tongue squamous cell carcinoma. Sci Rep. 2020;10(1):7877.

4. Yoon AJ, Wang S, Kutler DI, Carvajal RD, Philipone E, Wang T, Peters SM, LaRoche D, Hernandez BY, McDowell BD, Stewart CR, Momen-Heravi F, Santella RM. MicroRNA-based risk scoring system to identify early-stage oral squamous cell carcinoma patients at high-risk for cancer-specific mortality. Head Neck. 2020;42(8):1699-712.

5. Viet CT, Yu G, Asam K, Thomas CM, Yoon AJ, Wongworawat YC, Haghighiabyaneh M, Kilkuts CA, McGue CM, Couey MA, Callahan NF, Doan C, Walker PC, Nguyen K, Kidd SC, Lee SC, Grandhi A, Cheng AC, Patel AA, Philipone E, L. RO, Allen CT, and B.E. Aouizerat The REASON Score: An Epigenetic and Clinicopathologic Score to Predict Risk of Poor Survival in Patients with Early Stage Oral Squamous Cell Carcinoma 2021. Unpublished data.

6. Poage GM, Houseman EA, Christensen BC, Butler RA, Avissar-Whiting M, McClean MD, Waterboer T, Pawlita M, Marsit CJ, Kelsey KT. Global hypomethylation identifies Loci targeted for hypermethylation in head and neck cancer. Clin Cancer Res. 2011;17(11):3579-89.

7. Viet CT, Jordan RC, Schmidt BL. DNA promoter hypermethylation in saliva for the early diagnosis of oral cancer. J Calif Dent Assoc. 2007;35(12):844-9.

8. Viet CT, Schmidt BL. Methylation array analysis of preoperative and postoperative saliva DNA in oral cancer patients. Cancer Epidemiol Biomarkers Prev. 2008;17(12):3603-11.

9. Guerrero-Preston R, Michailidi C, Marchionni L, Pickering CR, Frederick MJ, Myers JN, Yegnasubramanian S, Hadar T, Noordhuis MG, Zizkova V, Fertig E, Agrawal N, Westra W, Koch W, Califano J, Velculescu VE, Sidransky D. Key tumor suppressor genes inactivated by "greater promoter" methylation somatic mutations in head neck cancer Epigenetics. 2014;9(7):1031-46.

10. Ha PK, Califano JA. Promoter methylation and inactivation of tumour-suppressor genes in oral squamous-cell carcinoma. Lancet Oncol. $2006 ; 7(1): 77-82$.

11. Huang MJ, Yeh KT, Shih HC, Wang YF, Lin TH, Chang JY, Shih MC, Chang JG. The correlation between CpG methylation and protein expression of P16 in oral squamous cell carcinomas. Int J Mol Med. 2002;10(5):551-4.

12. Shaw RJ, Hall GL, Woolgar JA, Lowe D, Rogers SN, Field JK, Liloglou T, Risk JM. Quantitative methylation analysis of resection margins and lymph nodes in oral squamous cell carcinoma. Br J Oral Maxillofac Surg. 2007;45(8):617-22.

13. Shaw RJ, Liloglou T, Rogers SN, Brown JS, Vaughan ED, Lowe D, Field JK, Risk JM. Promoter methylation of P16, RARbeta, E-cadherin, cyclin A1 and cytoglobin in oral cancer: quantitative evaluation using pyrosequencing. Br J Cancer. 2006;94(4):561-8.

14. Smiraglia DJ, Smith LT, Lang JC, Rush LJ, Dai Z, Schuller DE, Plass C. Differential targets of CpG island hypermethylation in primary and metastatic head and neck squamous cell carcinoma (HNSCC). J Med Genet. 2003;40(1):25-33.

15. Yeh KT, Shih MC, Lin TH, Chen JC, Chang JY, Kao CF, Lin KL, Chang JG. The correlation between CpG methylation on promoter and protein expression of E-cadherin in oral squamous cell carcinoma. Anticancer Res. 2002;22(6C):3971-5.

16. Li YF, Hsiao YH, Lai YH, Chen YC, Chen YJ, Chou JL, Chan MW, Lin YH, Tsou YA, Tsai MH, Tai CK. DNA methylation profiles biomarkers of oral squamous cell carcinoma Epigenetics. 2015;10(3):229-36.

17. Shu C, Zhang X, Aouizerat BE, Xu K. Comparison of methylation capture sequencing and Infinium MethylationEPIC array in peripheral blood mononuclear cells. Epigenetics Chromatin. 2020;13(1):51.

18. Lydiatt WM, Patel SG, O'Sullivan B, Brandwein MS, Ridge JA, Migliacci JC, Loomis AM, Shah JP. Head and Neck cancers-major changes in the American Joint Committee on cancer eighth edition cancer staging manual. CA Cancer J Clin. 2017;67(2):122-37.

19. Taylor JM, Ankerst DP, Andridge RR. Validation of biomarker-based risk prediction models. Clin Cancer Res. 2008;14(19):5977-83. 
20. Teh AL, Pan H, Lin X, Lim YI, Patro CP, Cheong CY, Gong M, Maclsaac JL, Kwoh CK, Meaney MJ, Kobor MS, Chong YS, Gluckman PD, Holbrook JD, Karnani N. Comparison of Methyl-capture Sequencing vs. Infinium 450K methylation array for methylome analysis in clinical samples. Epigenetics.

2016;11(1):36-48.

21. Heiss JA, Brennan KJ, Baccarelli AA, Tellez-Rojo MM, Estrada-Gutierrez G, Wright RO, Just AC. Battle of epigenetic proportions: comparing Illumina's EPIC methylation microarrays and TruSeq targeted bisulfite sequencing. Epigenetics. 2020;15(1-2):174-82.

22. Arantes L, De Carvalho AC, Melendez ME. and A. Lopes Carvalho, Serum, plasma and saliva biomarkers for head and neck cancer. Expert Rev Mol Diagn. 2018;18(1):85-112.

23. Nishitani S, Parets SE, Haas BW, Smith AK. DNA methylation analysis from saliva samples for epidemiological studies. Epigenetics. 2018;13(4):352-62.

\section{Figures}
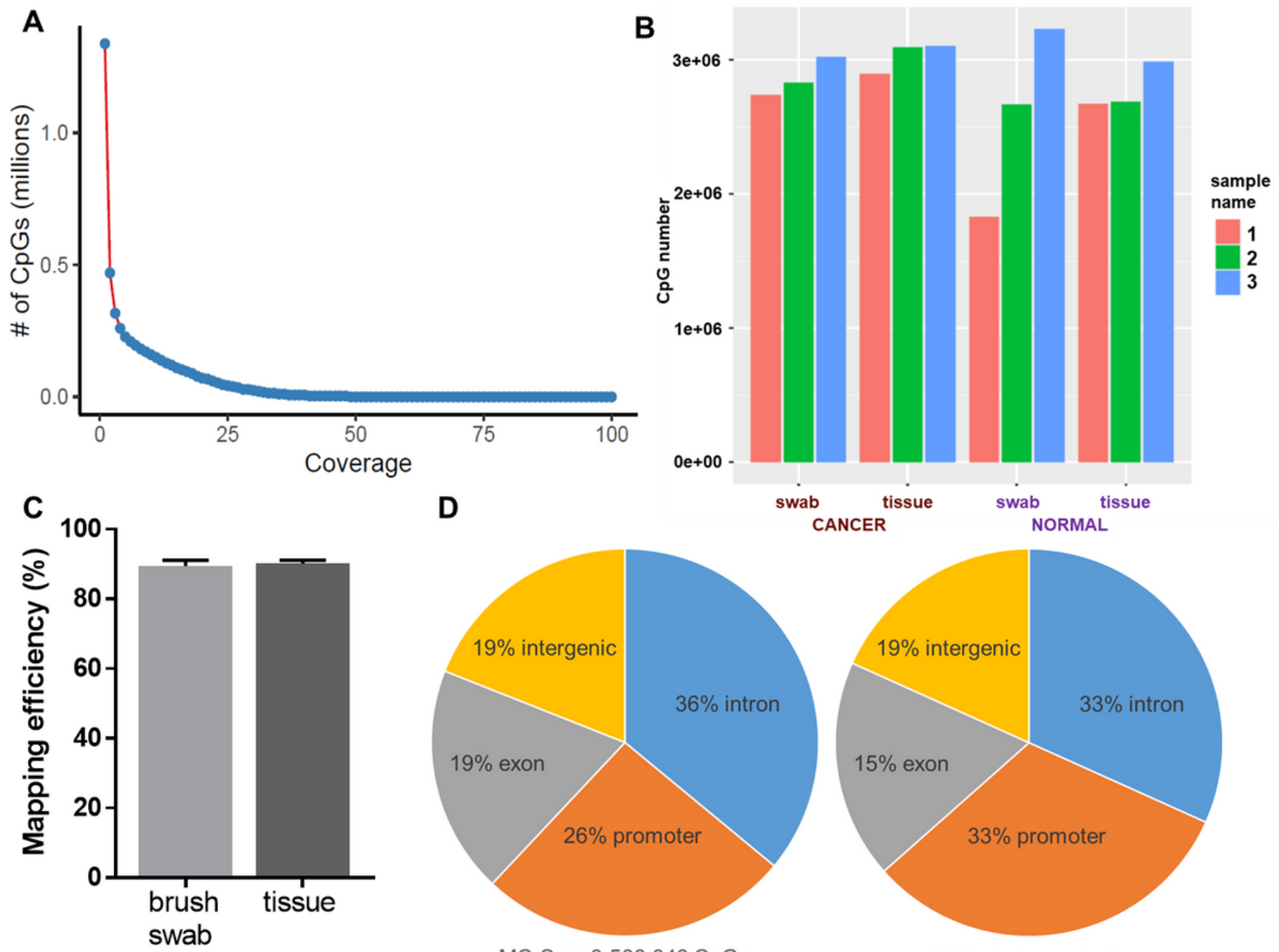

D

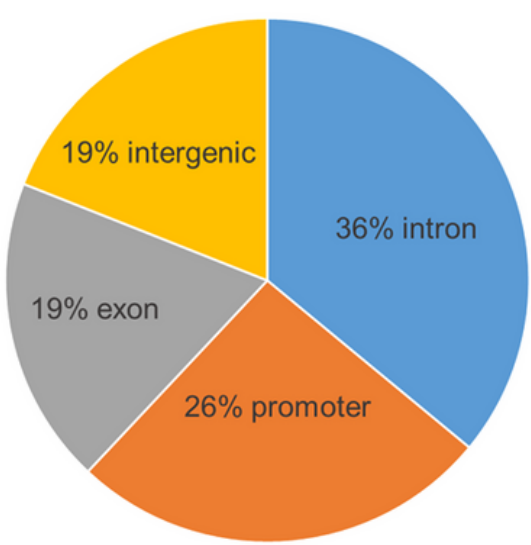

MC-Seq: $3,566,843 \mathrm{CpGs}$

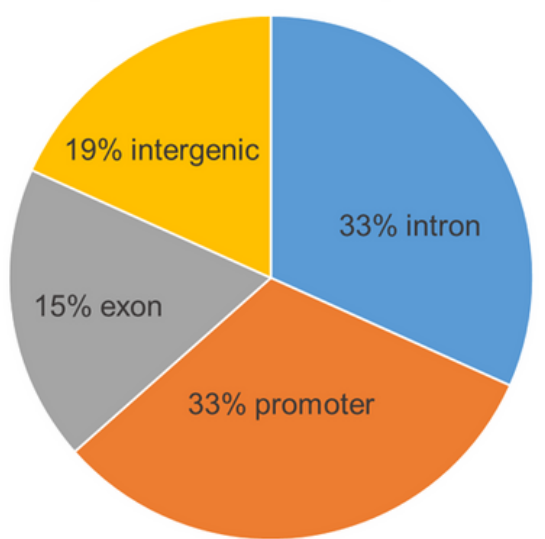

EPIC: 484,697 CpGs

\section{Figure 1}

(A) We compared depth of coverage in all CpGs and determined an inflection point at 10x coverage. (B) Using 10x read depth as a cutoff, we determined the number of quantified CpG sites in each sample. Average number of quantified CpGs meeting our criteria was 2,716,674 for swab samples and 2,904,261 for tissue samples, with no significant difference between the two sample types. (C) The average mapping efficiency was $89.45 \%$ for brush swabs and $90 \%$ for tissues, with no significant difference between the two sampling methods. (D) The pie charts detail the relative genic locations of the CpGs profiled by MC-Seq (left) and CpGs covered by the EPIC array that were profiled (right). MC-Seq provided more robust coverage of functional gene regions than the EPIC array. 

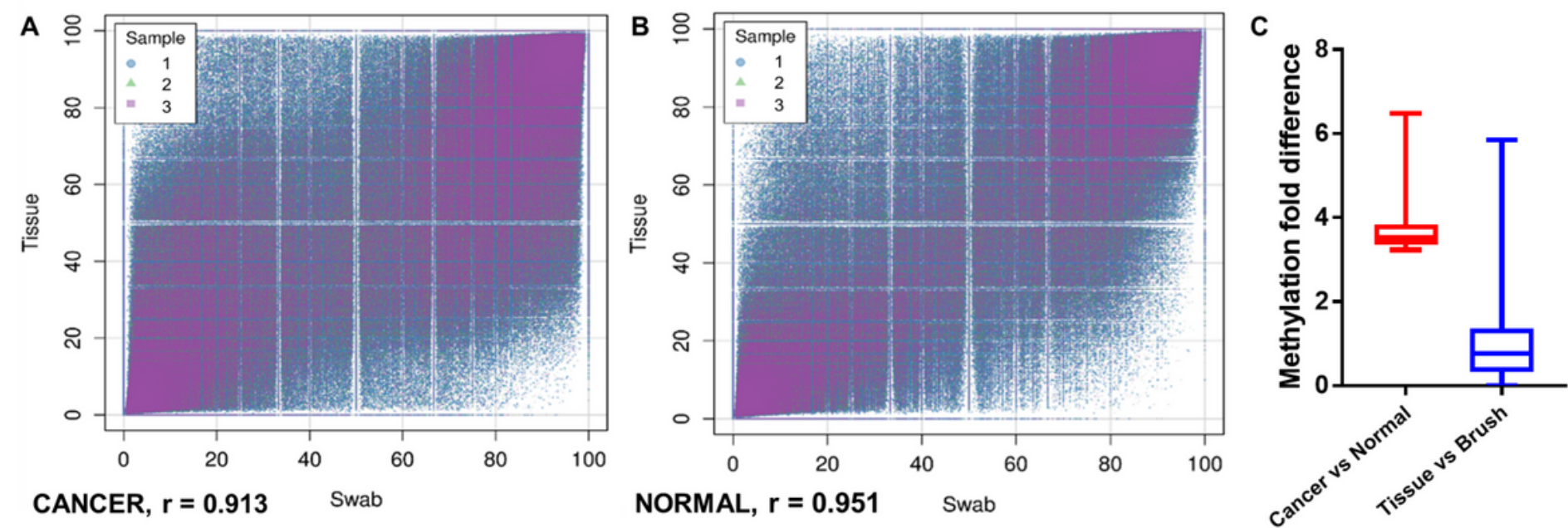

\section{Figure 2}

The scatterplots demonstrate the correlation between tissue and brush swab biopsies for (A) cancer and (B) normal sites of the 3 patients. The correlation values are noted. (C) By focusing on the top 1,000 most variable methylation features between cancer and normal samples quantified with MC-Seq, we determined the methylation difference between different sample types, visualized using box plots (median, quartiles, maximum and minimum whiskers). The p-values for each test of difference in CpG methylation by t-test were expressed as -log10(p-value), which had a median of 3.67 (i.e., p=0.00021) between cancer vs. normal. The same $\mathrm{CpG}$ sites were not differentially methylated [average - $\log 10$ ( $p$-value) $=0.96$ (i.e., $p=0.11$ )] between tissue vs. brush swabs, suggesting that brush swabs are a viable surrogate of tissue biopsy. 